\section{Substance Use among Muslims Residing in the United States: A Literature Review}

\author{
Sarah Ahmed and Nick Doukas* \\ Department of Concurrent Addiction Inpatient Treatment Service, Centre \\ for Addiction and Mental Health, Toronto, Ontario, Canada
}

growing population and the concern for the concerning rates of substance use has fostered an accumulation of research in this area, but there has yet to be a comprehensive review of the literature that focuses on substance use among Muslims in the United States.

This paper reviews the literature to retrieve and examine information available on substance use and the Muslim population. The review is divided into subsections based on the different areas of interest among researchers (college students, acculturation and treatment services). The paper ends with a discussion that describes the findings and makes recommendations for future research.

\section{Literature Review Search Method}

The databases Medline, PyscINFO, Cochrane CDSR, Cochrane CCTR, PsycArticles full text, ERIC, ProQuest, OVID, PubMed and EBSCO were searched from 1967 to the present year of 2016. The subject headings for Islam*, Arab or Muslim* specific to the database being searched were used. These search results were then combined with terms related to substance use. Keywords such as opium, hashish, and cannabis were searched in title or phrases such as Marijuana smoking, Marijuana abuse, Substance-Related Disorders were searched in the abstract field. In addition, the reference lists of all relevant articles were reviewed to identify articles that may have been missed in the database search. Furthermore, the following ethno cultural specific journals were reviewed to identify any further articles that may add to the literature review: Journal of Immigrant Minority Health, Journal of Muslim Mental Health and Journal of Ethnicity in Substance Abuse. This search generated six studies that focused on substance use among Muslim groups from the US.

\section{Cultural differences in the Islamic world}

While this paper reviews the literature on Muslims who reside in the US, it does not encompass the diversity of cultures and ethnicities of individuals that follow Islam. Regardless of where one chooses to call home, Muslims come from different parts of the world will have diverse cultures even though they share the same religion. For many Muslims, as with people of other faiths, their cultures play a strong role in their lives. As Islam spread beyond the Middle East into many different regions, ranging from China to Bosnia to Zanzibar, it came to be understood in many different cultural ways. This diversity was the result of the core set of religious beliefs interacting in complex ways with the many different contexts including history, cultural traditions, social, economic, political structures and geography and physical location in the world [9].

Islam involves peoples of many different races, ethnicities and cultures, many literatures and languages, with many histories and a myriad of interpretations some of which may conflict with each other. Islamic practices and beliefs are those that have roots in the Quran (which Muslims believe to be the last and unchanged revelation from God) and the Sunnah (traditions) of Prophet Muhammad (peace be upon him). Despite the multitude of cultural differences in Muslim majority countries, one rule is universal - any practice among Muslim-majority countries, which does not go back to the Quran or the Sunnah, is not considered to be an Islamic practice. It

\footnotetext{
Received: June 22, 2016; Accepted: September 20, 2016; Published: October 04, 2016

*Corresponding author: Nick Doukas, Department of Concurrent Addiction Inpatient Treatment Service, Centre for Addiction and Mental Health doukasnick@hotmail.com

Citation: Doukas N, Ahmed S (2016) Substance Use among Muslims Residing in the United States: A Literature Review. J Alcohol Drug Depend Subst Abus 2: 006 .
} 
is for this reason that substance use among Muslims is a very controversial topic and one that is often avoided.

\section{Islamic teaching on substance use}

There is a strict prohibition against substance use in Islam [10]. This is cited in verse 90 of Surah (chapter) Al Maidah of the Quran which states "O you who have believed, indeed, intoxicants, gambling, [sacrificing on] stone alters [to other than Allah], and divining arrows are but defilement from the work of Satan, so avoid it that you may be successful" (Quran 5:90). In Islam, the Quran and the prophetic traditions or the Sunnah are the central sources of references for principles that guide a Muslims way of life. Central to the teachings of Islam are preserving and protecting mankind and steering one away from harm and destruction. Islamic scholars agree that any substance that causes intoxication and harm to the body is not permissible and considered haram (forbidden) [11]. It is clear in the teachings that any substance that can cause harm to the body is strictly forbidden, newer substances, such as cigarette smoking have become widely accepted across cultures is also haram. While there is no direct reference to prohibition of cigarette smoking, the ruling on substances that cause harm to the body also applies to cigarette smoking. Therefore, one of the main fundamental concepts of Islam is protecting and promoting life and good health in all circumstances in all stages of life [11].

\section{Substance use among Muslim college students}

When searching for available studies conducted on substance use and the Muslim population, there is a growing trend that points to college students as an area of interest. It is estimated that $75 \%$ (10 million) of all American high school students have used tobacco, alcohol, marijuana or cocaine, and $46 \%$ (6.1 million) of these students continue to use substances [12]. One specific minority group of individuals that has received attention as a sub-group is Muslim college students. Much of the research conducted on substance use among Muslim college students has focused on understanding how risk factors and protective factors have an influence on risky behaviours. For example, Abu-Ras et al., [4] conducted secondary data analysis by obtaining estimates and examining risk factors, from the public access database from the 2001 Harvard School of Public Health College Alcohol Study. This study surveyed 119 colleges where students were selected to be a representative sample of accredited 4 -year colleges and universities in 38 states. Out of 10,401 students, $1.3 \%$ (135) reported to have been raised as Muslims. Authors reviewed the risk and protective factors associated with drinking such as religiosity, parental abstinence, parental disapproval of drinking, living at home with parents and lack of involvement in collegiate sports. Approximately $46.6 \%$ of Muslim students reportedly consumed alcohol in the past year, compared to $80.7 \%$ of their non-Muslim counterparts. All of the $46.6 \%$ reported at least one episode of binge drinking in the past year. The highest rates reported among these variables that influenced either alcohol use or abstinence were religiosity as a protective factor and parental approval of alcohol consumption as a risk factor.

Although alcohol consumption among US Muslim college students was lower compared to other faiths [4], the US Muslim rates are higher when compared to students in predominantly Muslim countries, specifically Egypt [13] and Jordan [14]. In Egypt, Refaat [13] conducted a descriptive cross-sectional survey-based study among a randomly selected sample of 687 college students in Ismailia to examine awareness and practice of risk behavior among Egyptian university students. Their results found that 121 students (18\%) engaged in risk behavior such as tobacco, substance and unprotected sexual activity. In Jordan, Suleiman, Shareef, Kharabsheh and Danoon [14] conducted a cross-sectional randomized survey-based study of 5064 university and college students to examine the impact of substance use among students and their attitude towards substance use. Twelve percent of students reported using alcohol and $29 \%$ reported using tobacco. It was observed that substance use was significantly higher among males. Risk factors that contributed towards drug use, as reported by the students, were acceptance of substance use, peer pressure and lack of communication with family. Despite having a lower consumption rate by US standards, in 2001, the rate of alcohol consumption among this population was still higher than that reported by students in the predominantly Muslim countries of Egypt [13] and Jordan [14].

In the second study from the US, Ahmed et al., [6] and colleagues used secondary analysis to look at discouraged or prohibited Islamic behaviours, such as substance use, gambling, tobacco use and non-marital sexual intercourse. These researchers reviewed data collected from 10,401 students who completed the US 2001 national college survey. Of this sample, $135(1.7 \%)$ were identified to be Muslims. Among this sub-group, $46.2 \%$ of the students reported to use alcohol, $24.6 \%$ engaged in illicit drug use, $37.3 \%$ smoked cigarettes, $30.4 \%$ gambled and $53.8 \%$ of students engaged in pre-marital sex. This study reports that $58.5 \%$ of Muslim students engage in at least one risk behavior of which $77.6 \%$ engaged in two or more risky behaviors [6].

In the third study that shows a continued trend in examining the risk and protective factors of Muslim students, Abu-Ras et al., [5] conducted a respondent-driven, survey-based study that was completed by 156 self-identified Muslim students in one university. Results of this study showed that $91.6 \%$ of this sample associated religiosity, negative experiences of alcohol use and proscriptive social influences with lifelong abstinence. Out of this sample $65 \%$ of life long abstainers identified religious beliefs to play a strong component in their life.

The three US studies found in this review that focus on college students-suggest that any prevention services geared towards Muslim students must address the entire family and diversity must be taken into account as Muslims can vary in levels of acculturation, socioeconomic backgrounds and degree of religious practice. When one considers that US Muslim students are a religious minority who may also have to navigate through a myriad of social, religious and cultural expectations in addition to other stressors such as discrimination [15], it is of no surprise that acculturation is another topic that has received attention among scholars.

\section{Substance use and acculturation in the United States}

Acculturation is a commonly occurring phenomenon in the US that has shown increasing attention among scholars [16]. Berry [17] defines acculturation as the change that occurs when a culturally distinct individual or group comes into contact with another culture. It is multidimensional and an individual can internalize more than one culture. While much of the literature on acculturation and substance use comes from the US and largely focused on Latino and Asian minorities, researchers are starting to notice a need to include Muslim Americans in acculturation studies. Much of this growing interest is due to the rapidly increasing Muslim population in the US, who are acculturating to the western practice of consuming mind a ltering substances [18]. 
Several writers exploring US acculturation assert that substance use can be problematic among individuals who identify with prohibitive cultures, such as that of most Arab countries [19-22]. Researchers have observed that individuals belonging to ethnic groups which prohibit the consumption of drugs and alcohol may have their protective factors reduced against substance use if acculturating in an alcohol and drug tolerant culture [18]. Arfken, Kubiak and Farrag [18] state that increased substance use among Arab Americans increases with acculturation possibly because of increased access to substances over time, more acceptance of substance use, or the use of substances to cope with discrimination.

The literature in this area has produced two studies that investigate correlations among Arab Americans and substance use. In the first study, Arfken [23] reviewed medical charts on 156 Arab American male clients, in one Michigan clinic, which showed that increased US acculturation was correlated with polysubstance abuse. The results also uncovered that de-acculturation from origin and home country cultures were also associated with substance use. Individuals in the sample with strong ties to their families, cultural and religious practices reported to experience more discrimination from the society leading to greater acculturation stress [23]. A reason for this could be due to the conflicting practices with mainstream culture, thereby increasing risk of alienation [7].

In the second study, Arfken, Arnetz, Fakhouri, Ventimiglia and Jamil [24], conducted a secondary data analysis on existing surveys to study alcohol use among Arab Americans through an acculturation lens. Overall it was reported that on a national level, 50.8\% of English speaking Arab Americans reported lifetime alcohol use compared to the White majority group whose lifetime alcohol consumption was 87\%. The rate of alcohol abuse among Arab Americans was 3.8\%, while $7.9 \%$ of the majority group reported alcohol abuse. However, on a state based level, English speaking Arab Americans had a similar rate of binge drinking as the White majority group, at approximately $17 \%$. Authors identified the lack of databases present to estimate alcohol use among Arab Americans, which limits an accurate understanding of prevalence rates. Based on the data they analyzed, alcohol consumption is consistent with an acculturation model.

Much of the literature provides readers with evidence to show the strong association between acculturation and substance use. This supports the need to develop culturally competent treatment services to ethnic minorities experiencing substance use concerns.

\section{Culture specific treatment services}

As reported earlier, Islam is the fastest growing religion in the world and the number of American Muslims is changing the ethnic makeup of the United States, which will affect various institutions such as publicly funded alcohol/other drug abuse. According to the 2004 US National Survey of Substance Abuse Treatment Services, among 13,011 services that facilitate treatment for substance use, only $26 \%$ offered Spanish and $0.3 \%$ offered Arabic [25]. This lack of culturally specific treatment for substance use has prompted another area of interest among researchers, which yielded one study in this review of the literature. Arfken et al., [26] conducted a secondary analysis using a sample of 69,989 that compared substance use treatment entry admissions in the US It was found that when compared to other ethnicities, Arab Americans reported to account for a small percentage of admissions to public funded alcohol/substance use treatment centers (0.3\%), but noticed that Arab Americans were entering treatment after a shorter duration of use. This study also found that almost all (99.1\%) of publicly funded treatment centers in the US did not alternate primary languages, which excludes individuals (immigrants, refugees) whose primary language is not English. This indicates that current treatment providers do not feel pressured to adopt a more cultural sensitive framework to expand language capabilities in their services [26].

Data from future research or from the studies retrieved in this review can inform the development of treatment programs and outreach efforts to provide a more appropriate provision of care for Muslim groups in North America and abroad.

\section{Discussion}

After a comprehensive review of the literature it is clear that research has recently begun to accumulate that examines substance use among Muslim in the US Among the six studies found in this review, Muslim college students received the most attention, with researchers examining risk and protective factors of alcohol use patterns and discouraged and prohibitive Islamic behaviors, such as substance use, gambling and pre-marital sexual intercourse [4-6]. Acculturation received the attention of two studies, which investigated the correlation between acculturation and substance use and the second one examining alcohol use patterns among US Arabs while being examined through the use of an acculturation lens [18,24]. In the final section of this paper the review yielded one study that focused on comparing substance use treatment admissions among various ethnicities and agencies in the US that provide non-English speaking accommodations for their clientele.

There was a strong indication among the studies found in where writers $[4-6,18,28]$ discussed the need to improve research methods. Furthermore, three out of the five studies found collected data that was either from only one clinic [18] one university [5] and from one state that had a high density of Muslim Arab Americans $[5,18,26]$ which would limit the generalizability of the findings. This limited generalizability is especially true for the three college student studies from the US because they are conducted by the same research group who may have used the same data set as well.

Data from these five studies was collected from individuals who followed through with accessing treatment, which excludes individuals that do not disclose substance use concerns due to the stigma. Another major limitation is the lack of data on female Muslim clients quite possibly because there is a stronger stigma associated with substance use among Arab-American women when compared to their male counterparts [27].

Several researchers have commented that study methods could be improved by including the measurement of variables such as religion, ethnicity, culture, societal norms and influences $[28,29]$. Among the five studies that were reviewed, no qualitative studies were found. One of the major strengths of qualitative methods is the knowledge it provides through the dynamics of social process [30] and its distinct ability to answer how and why questions [31]. While qualitative studies lack generalizability, they are tempered by the emergence of comparable findings within diverse samples and settings [32]. It will also be challenging to collect face to face interview data due to the stigma associated with substance use among individuals that identify with a prohibitive religion/culture.

Research on substance use among US Muslims has likely been conducted because of the rapidly growing population and evidence showing a significant concern regarding substance use consumption. 

006.

Services tailoring to this group's treatment need will have to seriously consider allocating some funding toward culturally specific services as well as culturally competent training for staff. But this will not be easy when considering the diversity of cultures that exist among this population.

\section{Conclusion}

In conclusion, more research is needed on a global level to meet the diverse nature of a religion that encompasses many cultures across many countries. This is also true about the diversity of Muslims that reside in the United States. This stresses the need to include larger sample sizes and find ways to include women. Researchers can also improve methods such as identifying sub-groups and variables that are significant to this population. The literature describes a rising need for culturally diverse treatment services to address substance use among this group because the ethnic landscape is changing and the growing concern for treatment demands attention. Several factors play a role on substance use and treatment, such as acculturation and language barriers. Program developers will have to be cognizant of all such factors in order to create an appropriate provision of care that is welcoming to treatment seeking Muslims and retains them as well.

\section{References}

1. Nimer M (2003) The North American Muslim resource guide: Muslim community life in the United Nimer States and Canada. Choice Reviews Online 40.

2. PEW Research Center (2011) The Future of the Global Muslim Population. PEW Research Center, Washington, DC, USA.

3. PEW Research Center (2015) The Future of World Religions: Population Growth Projections, 2010-2050, PEW Research Center, Washington D.C., USA.

4. Abu-Ras W, Ahmed S, Arfken C (2010) Alcohol Use among U.S. Muslim College Students: Risk and Protective Factors. J Ethn Subst Abuse 9: 206-220.

5. Abu-Ras W, Ahmed S, Arfken C (2013) Respondent-driven sampling of Muslim undergraduate U.S. college students and alcohol use: Pilot study. Soc Psychiatry Psychiatr Epidemiol 48: 945-953.

6. Ahmed S, Abu-Ras W, Arfken C (2014) Prevalence of Risk Behaviors among U.S. Muslim College Students. Journal of Muslim Mental Health 8.

7. Amer M, Hovey J (2007) Socio-demographic differences in acculturation and mental health for a sample of 2 nd generation/early immigrant Arab Americans. J Immigr Minor Health 9: 335-347.

8. D’Agostino M (2003) Muslim personhood: Translation, transnationalism and Islamic religious education among Muslims in New York City. Journal of Muslim Minority Affairs 23: 285-294.

9. Muslim Histories \& Cultures (2009) Seminar One: Sources of the Tradition. Muslim Histories \& Cultures.

10. Michalak L, Trocki K, Bond J (2007) Religion and alcohol in the U.S. National Alcohol Survey: How important is religion for abstention and drinking? Drug and Alcohol Dependence 87: 268-280.

11. Kamarulzaman A, Saifuddeen S (2010) Islam and harm reduction. Int J Drug Policy 21: 115-118.

12. CASA (2011) National Study Reveals: Teen Substance Use America's \#1 Public Health Problem, CASA, New York ,USA.

13. Refaat A (2004) Practice and awareness of health risk behavior among Egyptian university students. East Mediterr Health J 10: 72-81.
14. Suleiman R, Shareef M, Kharabsheh S, Danoon M (2003) Substance use among university and college students in Jordan. Arab Journal of Psychiatry 14: $94-105$

15. Ahmed S (2012) Adolescent and emerging adults. In: Ahmed S, Amer MM (eds.). Counseling Muslims: Handbook of mental health issues and interventions, Michigan Publishing, Michigan, USA.

16. Zane N, Kim JH (1994) Substance use and abuse. Confronting critical health issues of Asian and Pacific Islander Americans, USA. Pg No: 316-343.

17. Berry JW (2003) Conceptual approaches to acculturation. In: Chun KM, Organista PB, Marin G (eds.). Acculturation: Advances in Theory, Measurement, and Applied Research. American Psychological Association, Washington D.C., USA.

18. Arfken C, Kubiak S, Farrag M (2009) Acculturation and Polysubstance Abuse in Arab-American Treatment Clients. Transcult Psychiatry 46: 608-622.

19. Abudabbeh N, Nydell M (1993) Transcultural counseling and Arab-Americans. In: McFadden J (ed.), Transcultural counseling: Bilateral and international perspectives. American Counseling Association, Alexandria, USA. Pg No: $261-284$.

20. Beitin B, Allen KR (2005) Resilience in Arab American couples after September 11, 2001: A systems perspective. J Marital Fam Ther 31: 251-267.

21. Erickson C, Al-Timimi N (2001) Providing mental health services to Arab Americans: Recommendations and considerations. Cultur Divers Ethnic Minor Psychol 7: 308-327.

22. Hammoud M, White C, Fetters M (2005) Opening cultural doors: Providing culturally sensitive healthcare to Arab American and American Muslim patients. Am J Obstet Gynecol 193: 1307-1311.

23. Arfken C, Berry A, Owens D (2009) Pathways for Arab Americans to substance abuse treatment in Southeastern Michigan. Journal of Muslim Mental Health 4: 31-46.

24. Arfken C, Arnetz B, Fakhouri M, Ventimiglia M, Jamil H, et al. (2011) Alcohol Use among Arab Americans: What is the Prevalence? J Immigr Minor Health13: 713-718.

25. National Institute on Drug Abuse (2004) National Survey of Substance Abuse Treatment Services (N-SSATS), ICPSR04256-v5. United States Department of Health and Human Services, Substance Abuse and Mental Health Services Administration, Office of Applied Studies, National Addiction \& HIV Data Archive Program, National Insititute of Health, Michigan, USA.

26. Arfken C, Kubiak S, Farrag M (2008) Arab Americans in Publicly Financed Alcohol/Other Drug Abuse Treatment. Alcoholism Treatment Quarterly 26: 229-240.

27. Abudabbeh N, Hamid A (2001) Substance use among Arabs and Arab Americans. In: Straussner SLA (eds.). Ethnocultural factors in substance abuse treatment. Guilford Press, New York, USA. Pg No: 275-290.

28. Galen L, Rogers W (2004) Religiosity, alcohol expectancies, drinking motives and their interaction in the prediction of drinking among college students. J Stud Alcohol 65: 469-476.

29. Johnson T, Sheets V, Kristeller J (2008) Identifying Mediators of the Relationship between Religiousness/Spirituality and Alcohol Use. J Stud Alcohol Drugs 69: 160-170.

30. Bryman A (2006) Integrating quantitative and qualitative research: How is it done? Qualitative Research 6: 97-113.

31. Mason J (2006) Mixing methods in a qualitatively driven way. Qualitative Research 6: 9-25.

32. Nicholas DB, Globerman J, Antle BJ, McNeill T, Lach LM (2006) Processes of metastudy: A Study of Psychosocial Adaption to Childhood Chronic Health Conditions. International Journal of Qualitative Methods 5: 1-10. 